\title{
Notes on Transliteration and Pronunciation
}

For Tamil words, I use a somewhat simplified transliteration system sufficient to indicate the pronunciation of words, and thus the terms themselves (since no Tamil homonyms are used), for those familiar with Tamil diacritics in roman script. (I do not use diacritics to differentiate Tamil letters with identical or near-identical sounds.) Other readers may wish to use the pronunciation guide below.

For Hindi/Sanskrit words, I use standard transliterated spellings in English literature, usually without diacritics (such as bhakti, darshan) except for those words that are central to a portion of the analysis and therefore appear frequently (for example, $d \bar{a} n$ ).

For names of places, castes, deities, and people, I avoid diacritics and use one of the standard transliterations likely to be readily pronounceable for readers unfamiliar with Tamil, Hindi, or Sanskrit.

For readers unfamiliar with Tamil transliteration conventions, I offer this informal pronunciation guide to the letters that appear in Tamil words in this text.

Vowels:

$$
\begin{aligned}
& \text { a ("uh") } \\
& \bar{a} \text { ("ah") } \\
& \text { e ("eh") } \\
& \text { è (“ey" as in hey) } \\
& i \text { ("i" as in bin) } \\
& \overline{1} \text { ("ee") } \\
& \text { o ("o" as in or) } \\
& \text { o ("oh") } \\
& \text { u ("oo" as in book) } \\
& \text { ù ("oo" as in toot) } \\
& \text { ai ("ai" as in aisle) }
\end{aligned}
$$

Consonants:

Several consonants have both retroflex (back-of-the-palate) and dental (front-of-the-palate) forms. These consonants are in the dental form if 
they are unmarked (i.e., if they have no diacritic), and in the retroflex form if marked with a period-like diacritic underneath the letter (l, n, ț).

In addition, many consonants are pronounced differently depending on whether they occur at the beginning of a word, are doubled in the middle of a word, or occur singly in the middle of a word. "K," for example, is pronounced in a way familiar to English readers if it appears at the beginning or if it is doubled ("kk") in the middle of the word. If it appears as a single consonant in the middle, however, it is pronounced "g" or "h." (In phonological terms, these are unvoiced vs. voiced consonants.) Consonants that follow this rule are pronounced as below, with the initial or double consonant sound listed first and the single mid-word consonant sound listed second.

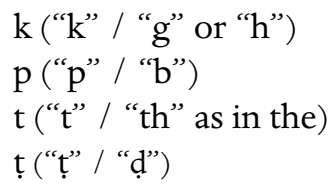

Other consonants:

$\mathrm{c}$ is pronounced " $\mathrm{s}$ " or "ch" when it falls at the beginning of a word or is doubled, " $s$ " when surrounded by vowels, and " $\mathrm{j}$ " following $n$ (i.e., nc = "nj")

1 is pronounced either like the retroflex l, or a back-of-the-mouth "standard" English r, or somewhere in between, depending on the speaker

$\dot{\mathrm{n}}$ ("ng" as in sing)

ș ("sh" as in wash)

Finally, certain consonants $(\mathrm{d}, \mathrm{j}, \mathrm{s})$ indicate a "loan word" borrowed from another Indian language, and are pronounced in a way familiar to English speakers. 


\section{LIVING CLASS IN URBAN INDIA}


